Was ist neu in der medika-
mentösen Therapie?
Wir halten Sie auf dem
Laufenden mit Berichten von Kon-
gressen und Symposien der phar-
mazeutischen Industrie.

\title{
Zulassungserweiterung für niedermolekulares Heparin
}

\section{Auch internistische Patienten brauchen Thromboseschutz}

- Jeder immobilisierte Patient hat ein erhöhtes Thromboserisiko. Das gilt besonders für akut erkrankte internistische Patienten, die durch höheres Alter, frühere Thromboembolien, Tumorleiden, chronische Herzinsuffizienz, COPD, Übergewicht usw. häufig ohnehin thromboseanfällig sind. Eine wirksame und unkomplizierte Thromboseprophylaxe lässt sich bei diesen Patienten mit Certoparin (MonoEmbolex ${ }^{\circledast}$ ) durchführen. Das niedermolekulare Heparin (NMH), das unabhängig vom Risiko in fixer Prophylaxedosierung gegeben wird, hat im März eine entsprechende Zulassungserweiterung erhalten.

Damit ist Certoparin jetzt nicht nur im Zusammenhang mit Operationen und nach akutem ischämischem Schlaganfall, sondern auch bei nicht chirurgischen $\mathrm{Pa}$ tienten mit erhöhtem Risiko für venöse Thromboembolie (VTE) und Immobilisation aufgrund einer akuten Erkrankung (z. B. akute Atemwegs-, Herz- oder Gefäßerkrankung, gastrointestinale oder neurologische Erkrankung) zur VTE-Prophylaxe einsetzbar.

\section{CERTIFY-Studie spricht für NMH}

Grundlage für die Indikationserweiterung von Certoparin war die CERTIFY-Studie, die Prof. Dr. Hanno Riess, Berlin, präsentierte. An der randomisierten Doppelblindstudie waren 3239 akut erkrankte hospitalisierte internistische Patienten über 70 Jahren mit relevanter Einschränkung der Mobilität beteiligt. Sie erhielten im Mittel für neun Tage entweder Certoparin (3000 I.E. anti-Xa $1 \times$ täglich plus zwei Placeboinjektionen) oder unfraktioniertes Heparin (UFH, 5000 I.E. $3 \times$ täglich) subkutan. Mit Kompressionssonografie wurde nach Thrombosen gefahndet.

Der primäre Endpunkt, bestehend aus proximaler tiefer Beinvenenthrombose, nicht tödlicher Lungenembolie und Tod im Zusammenhang mit VTE, trat unter Certoparin bei 3,9\% der Patienten auf, unter UFH bei $4,5 \%$. Blutungen waren in der NMH-Gruppe signifikant seltener als in der UFH-Gruppe (3,2\% vs. 4,6\%). Damit war das Studienziel, die Nichtunterlegenheit des NMH gegenüber dem alten Standard UFH zu beweisen, erreicht.

\section{Unterschätzte Gefahr}

Drei von vier Patienten, die eine VTE erleiden, sind nicht chirurgische Patienten! Trotzdem werden internistische Patienten im Alltag immer noch als nicht so gefährdet wahrgenommen, monierte Riess. Der Einsatz der medikamentösen Thromboseprophylaxe in der inneren Medizin bleibe deutlich hinter den Vorgaben der Leitlinien zurück.

In der S3-Leitlinie der AWMF zur Prophylaxe venöser Thromboembolien heißt es: „Stationäre Patienten mit akuten internistischen Erkrankungen und Bettlägerigkeit sollen eine medikamentöse VTE-Prophylaxe erhalten." Laut Riess benötigt jeder internistische Patient, der stationär aufgenommen wird und den Tag überwiegend im Bett verbringt, eine solche Prophylaxe, sofern er keine Kontraindikationen aufweist.

\footnotetext{
- Dr. med. Ulrike Wepner

Quelle: Industriesymposium, GTH-Jahres-

tagung, Nürnberg, 24./26. Februar 2010 (Veranstalter: Novartis Pharma $\mathrm{GmbH}$ )
}

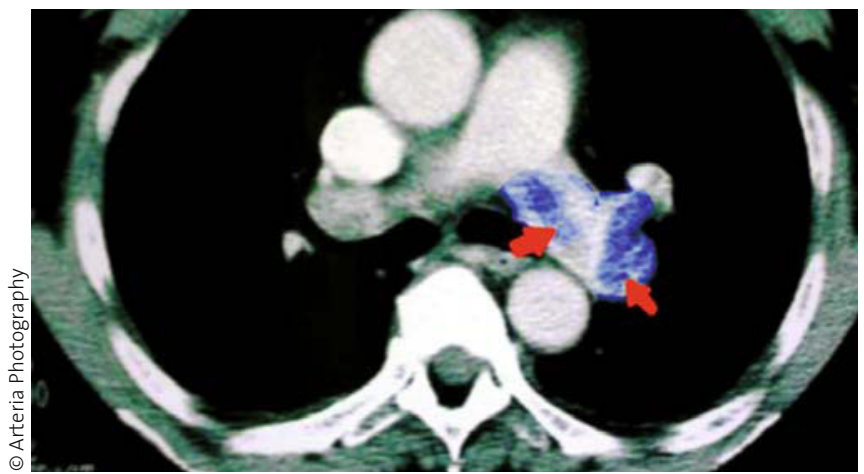

Thrombus in der linken Lungenarterie.

\section{Das empfiehlt die S3-Leitlinie}

- Stationäre Patienten mit akuten internistischen Erkrankungen und Bettlägerigkeit sollen eine medikamentöse VTE-Prophylaxe erhalten. $\uparrow \uparrow$

- Die medikamentöse Prophylaxe soll vorzugsweise mit einem NMH in Hochrisikoprophylaxe-Dosierung oder mit Fondaparinux erfolgen. $\uparrow \uparrow$

- Bei Kontraindikationen gegen die medikamentöse VTEProphylaxe sollten medizinische Thromboseprophylaxestrümpfe eingesetzt werden. $\uparrow$

- Die medikamentöse Prophylaxe sollte in der Regel für 6-14 Tage durchgeführt werden. $\uparrow$ 